\title{
Co-activation of Hedgehog and Wnt signaling pathways is associated with poor outcomes in triple negative breast cancer
}

\author{
KIMBERLY M. ARNOLD ${ }^{1,2}$, RYAN T. POHLIG ${ }^{1}$ and JENNIFER SIMS-MOURTADA ${ }^{1-3}$ \\ ${ }^{1}$ Center for Translational Cancer Research, Helen F. Graham Cancer Center and Research Institute, Newark, DE 19713; \\ Departments of ${ }^{2}$ Medical Laboratory Sciences and ${ }^{3}$ Biological Sciences, University of Delaware, Newark, DE 19716, USA
}

Received March 17, 2017; Accepted August 3, 2017

DOI: $10.3892 / 01.2017 .6874$

\begin{abstract}
Hedgehog $(\mathrm{HH})$ and Wnt pathway activation have been implicated in poor prognosis of breast cancer. Crosstalk between these two pathways has been demonstrated to be important in breast cancer progression, however the association between these two pathways and breast cancer survival rate is unknown. The present study comprised a cohort of 36 patients with triple negative breast cancer (TNBC) to investigate co-activation of $\mathrm{HH}$ and canonical Wnt pathway in association to patient outcome. All patients had varying degrees of cytoplasmic sonic $\mathrm{HH}$ and glioma-associated oncogene homolog (Gli)-1 staining, which positively correlated with tumor stage. Nuclear $\beta$-catenin was additionally correlated to tumor stage. A significant association was observed between nuclear Gli-1 and nuclear $\beta$-catenin. Co-activation of $\mathrm{HH}$ and Wnt pathways was associated with poorer prognosis in TNBC patients resulting in a greater risk of early recurrence and decreased overall survival rate compared with patients with only one pathway activated. Therefore, the combined activation status of the $\mathrm{HH}$ and Wnt pathways may be a useful prognostic marker for TNBC patients at risk for early recurrence.
\end{abstract}

\section{Introduction}

Triple negative breast cancer (TNBC) accounts for approximately $15 \%$ of all breast cancers. This subset of breast cancer is defined by the absence of positive staining for estrogen receptor (ER) and progesterone receptor (PR) and lack

Correspondence to: Dr Jennifer Sims-Mourtada, Center for Translational Cancer Research, Helen F. Graham Cancer Center and Research Institute, 9701 Ogletown Stanton Road, Suite 4300, Newark, DE 19713, USA

E-mail: jsimsmourtada@christianacare.org

Abbreviations: TNBC, triple negative breast cancer; $\mathrm{SHH}$, sonic Hedgehog; HH, Hedgehog

Key words: triple negative breast cancer, Hedgehog signaling, Wnt signaling, $\beta$-catenin amplification of HER2. Molecular profiling of TNBC has revealed that most TNBC have a subset of gene expression patterns that are associated to basal-myoepithelial cells in the breast. TNBC is more likely to affect younger women, women of African American descent, women who were exposed to radiation at an early age and BRCA1 mutation carriers (1-3). TNBCs are considered aggressive tumors with a high degree of genomic instability and therefore usually present as high grade, large tumors with a high proliferative index (4).

Due to the aggressive nature of TNBC tumors and decreased efficacy of targeted chemotherapy, TNBCs are associated with poor prognosis and early visceral metastasis (5). Survival rates for women who have a systemic or local recurrence within 3-5 years of treatment (early recurrence) are significantly lower survival rates for hormone receptor positive $\left(\mathrm{ER} / \mathrm{PR}^{+}\right)$breast cancer, while those who recur after 5 years have survival rates similar to $\mathrm{ER} / \mathrm{PR}^{+}$cancers (4). Understanding the molecular mechanisms that drive growth of TNBC may lead to better treatment, as well as predictive biomarkers that may identify women at risk for aggressive disease. TNBCs represent a collection of subtypes, some of which are associated with rapid progression, while others are reported as being less aggressive. Therefore, there is a clinical need to determine the molecular differences between these subtypes of TNBC patients in order to provide the most effective treatment; however a reliable prognostic marker has not been identified.

Molecular pathways crucial in embryonic development, including the Hedgehog $(\mathrm{HH})$ and Wnt signaling pathways have been shown to play an important role in breast cancer development and progression. Abnormal regulation of these pathways can lead to activation of genes essential for cell proliferation, cell survival, and therapeutic resistance $(6,7)$. In addition, activation of $\mathrm{HH}$ and Wnt signaling have been implicated in the growth and resistance of cancer stem cells and maintenance of the stem cell niche (8-12). These pathways are also key regulators of genes controlling epithelial-mesenchymal transition (EMT), contributing to cancer invasion and metastasis $(13,14)$.

The $\mathrm{HH}$ signaling pathway is critical for growth and differentiation during embryonic development. Initiation of the pathway requires secreted $\mathrm{HH}$ molecules [Sonic $\mathrm{HH}$ (SHH), Desert and Indian] to bind to and inhibit the cell surface $\mathrm{HH}$ receptor, Patched $(\mathrm{PTCH})$. This binding relieves the PTCH-mediated suppression of the transmembrane 
protein smoothened (SMO), leading to multiple intracellular events that result in the stabilization, nuclear translocation and activation of the glioma-associated oncogene (Gli) family of transcription factors, which initiate transcription of $\mathrm{HH}$ target genes (15). However, aberrant $\mathrm{HH}$ signaling has been shown to be associated with malignant transformation in many tissues, including breast (16-19). It has been reported that protein levels of SHH, PTCH, and Gli-1 in the breast tumor are significantly elevated compared to the adjacent normal breast ducts $(20,21)$. Overactivation of $\mathrm{HH}$ signaling is thought to result in increased number of mammary stem cells, resulting in tumor formation (10). However, there are a limited number of studies on how the $\mathrm{HH}$ pathway relates to patient prognosis. Inactivation of $\mathrm{HH}$ through high expression of Ptch in patients with invasive ductal breast carcinoma was associated with a favorable prognosis (22). Additionally, patients with inflammatory breast cancer had elevated SHH expression, which was associated with poor prognosis (23).

The Wnt signaling pathway is a complex signaling network involved in many physiological processes, including tissue patterning, cell migration, EMT, and maintenance of stem cells (24-26). Canonical Wnt signaling involves stabilization of $\beta$-catenin and translocation of the protein to the nucleus resulting in activation of Wnt target genes. In the absence of Wnt ligand, $\beta$-catenin is in a multi-protein, cytoplasmic complex with axin and adenomatous polyposis coli (APC). Upon phosphorylation by glycogen synthase kinase-3 (GSK-3 $\beta$ ) and casein kinase- $1 \beta, \beta$-catenin is degraded $(24,26,27)$. In the presence of canonical Wnt proteins, Wnt binds to the receptors, Frizzled and lipoprotein receptor-related proteins (LRP) 5 and 6 , resulting in activation of Dishevelled. This in turn disrupts the $\beta$-catenin-APC-axin complex, blocking degradation of $\beta$-catenin and allowing accumulation and translocation of $\beta$-catenin to the nucleus where it forms an activation complex with the T-cell factor/lymphoid enhancing factor (Tcf/LEF) family of transcription factors, leading to expression of genes critical for development of cell transformation and cancer, including cyclin D1, c-Myc, and peroxisome-proliferator-activated receptor- $\beta$ (PPAR- $\beta$ ) (26-28). Only canonical Wnt signaling drives $\beta$-catenin mediated transcription and thus nuclear $\beta$-catenin is considered an indicator of Wnt activation (29).

Aberrant canonical Wnt signaling has been shown in many tumors, including breast (9,30-32). In mouse models, overexpression of a $\beta$-catenin mutant and mutations of the $A p c$ gene have resulted in mammary tumorigenesis (33). Human breast cancer cell lines show higher amplification of canonical Wnt genes $(34,35)$ concomitant with downregulation of non-canonical Wnt gene s (35) and a higher expression of Dishevelled (36). Normally in the human breast duct and lobules, $\beta$-catenin is localized at the cell membrane, bound to E-cadherin and not part of Wnt signaling (25,31,33,37-39). Studies have reported a complete loss of $\beta$-catenin at the cell membrane in invasive lobular breast carcinomas consistent with a loss of E-cadherin; however, localization of $\beta$-catenin in the cell was not reported in these studies $(37,38)$. In addition, in invasive ductal carcinoma, a loss of membranous $\beta$-catenin correlates with increased expression of canonical Wnt signaling target genes, suggesting activation of the canonical Wnt pathway and this correlates with poorer outcomes in breast cancer patients (31). However, others have shown that a loss of membranous $\beta$-catenin staining is not correlated with tumor stage, grade, or outcome (37). Therefore, how activation of the $\mathrm{Wnt} / \beta$-catenin signaling pathway correlates with breast cancer patient prognosis is not completely clear.

Independently, $\mathrm{HH}$ and Wnt signaling pathways play a role in the progression of breast cancer and therefore the crosstalk between these developmental pathways is thought to provide tumor cells with multiple mechanisms to evade chemotherapy. Current research has shown a potential for crosstalk between $\mathrm{HH}$ and Wnt signaling in cancer. Co-activation of $\mathrm{HH}$ and Wnt signaling has been exhibited in a variety of cancers, including basal cell carcinoma and pancreatic ductal adenocarcinoma (40). In contrast, it has also been shown that the activity of the $\mathrm{Wnt} / \beta$-catenin pathway can be suppressed by players of the $\mathrm{HH}$ pathway $(41,42)$. However, the impact of $\mathrm{HH}$ and $\mathrm{Wnt}$ signaling crosstalk in breast cancer outcomes is poorly understood. Therefore, we wanted to determine if co-activation of the $\mathrm{HH}$ signaling pathway and canonical Wnt pathway could be used as a prognostic marker in TNBC patients.

\section{Materials and methods}

Patient population. Breast cancer tissue specimens were obtained from the biorepository at the Helen F. Graham Cancer Center and Research Institute (HFGCCRI) under protocol approved by the institutional review board. De-identified samples were obtained from surgical resection from women diagnosed with TNBC who consented to the use of their tissues for research. Tissue blocks were prepared by formalin-fixation and embedded in paraffin for serial sectioning. Each sample underwent a pathological diagnostic procedure including staining for the ER, PR and HER2 expression. HER2 status was further confirmed by FISH. Pathologically confirmed TNBC (ER expression <1.0\%) samples were used in this study. Hematoxylin and eosin (H\&E) stains of tumors sections were reviewed by a breast cancer pathologist to determine the percentage of tumor nuclei and necrosis. Only samples containing $>60 \%$ tumor nuclei were used for this study.

Immunohistochemical procedure. Immunohistochemical staining was performed on $4 \mu \mathrm{m}$ thick tissue sections of each specimen using the LSAB+System-HRP staining kit (Dako; Agilent Technologies, Inc., Santa Clara, CA, USA) according to the manufacturer's instructions as previously described (43). Antibodies to SHH (cloneEP1190Y; Abcam, Cambridge, UK; and H160-sc9026; Santa Cruz Biotechnology, Inc., Dallas, TX, USA), Gli-1 (Clone H-300; Santa Cruz Biotechnology, Inc.), and $\beta$-catenin (cloneE247; Abcam) were used at a dilution of 1:100 for SHH, 1:1,000 for Gli-1 and 1:500 for $\beta$-catenin. Both SHH antibodies showed a similar staining pattern. Specificity of staining was confirmed by omission of the primary antibody and staining with an isotype matched control antibody (Jackson Laboratory, Ben Harbor, ME, USA). Slides were scored by two independent investigators blinded to the sample data. Slides were scored as having no expression (0), weak (1), moderate (2), or strong (3) tumor cell staining. Nuclear staining was noted if nuclear stain was observed in more than $10 \%$ of cells in 3 fields at $x 40$ magnification. 
Table I. Patient characteristics and expression of HH and WNT pathway members.

\begin{tabular}{|c|c|c|c|c|c|}
\hline Characteristics & No. & $\%$ & P-value stage & P-value RFS & P-value OS \\
\hline \multicolumn{6}{|l|}{ Stage } \\
\hline $1 \mathrm{~A}$ & 6 & 16.7 & & & \\
\hline $1 \mathrm{~B}$ & 1 & 2.8 & & & \\
\hline $2 \mathrm{~A}$ & 14 & 38.9 & & & \\
\hline $2 \mathrm{~B}$ & 4 & 11.1 & & & \\
\hline $3 \mathrm{~A}$ & 4 & 11.1 & & & \\
\hline $3 B$ & 0 & 0.0 & & & \\
\hline $3 \mathrm{C}$ & 5 & 13.9 & & & \\
\hline 4 & 2 & 5.6 & & 0.0055 & 0.0036 \\
\hline \multicolumn{6}{|l|}{ Grade } \\
\hline 2 & 5 & 13.9 & & & \\
\hline 3 & 31 & 86.1 & 0.3 & 0.732 & 0.985 \\
\hline \multicolumn{6}{|l|}{$\mathrm{SHH}$} \\
\hline 1 & 8 & 22.2 & & & \\
\hline 2 & 20 & 55.6 & & & \\
\hline 3 & 8 & 22.2 & 0.0440 & 0.051 & 0.026 \\
\hline \multicolumn{6}{|l|}{ Gli-1 } \\
\hline 0 & 1 & 2.8 & & & \\
\hline 1 & 9 & 25.0 & & & \\
\hline 2 & 14 & 38.9 & & & \\
\hline 3 & 12 & 33.3 & 0.0010 & 0.0213 & 0.017 \\
\hline \multicolumn{6}{|l|}{ Nuclear Gli-1 } \\
\hline$<10 \%$ & 11 & 30.6 & & & \\
\hline$>10 \%$ & 25 & 69.4 & 0.0080 & 0.003 & 0.0387 \\
\hline \multicolumn{6}{|l|}{$\beta$-catenin } \\
\hline Membranous & 13 & 36.1 & & & \\
\hline Nuclear/cytoplasmic & 23 & 63.9 & 0.0030 & 0.019 & 0.025 \\
\hline
\end{tabular}

HH, Hedgehog; SHH, sonic HH; Gli-1, glioma-associated oncogene homolog-1; RFS; relapse-free survival; OS, overall survival.

Statistical analysis. Spearman correlations were used to test the relationships between $\mathrm{SHH}$, Gli-1, and $\beta$-catenin staining and clinical characteristics. Due to the small sample size, samples were grouped into three categories based on activation of $\mathrm{HH}$ and Wnt pathways for survival analysis: i) Those with activation of neither pathway (no staining for nuclear Gli-1 and nuclear $\beta$-catenin), $n=8$; ii) only one pathway activated, staining for either nuclear Gli-1 or nuclear $\beta$-catenin, $\mathrm{n}=8$; and iii) those with staining for nuclear expression of both pathways, $n=20$. These groups were then compared using chi-square tests for survival and recurrence. Lastly, Kaplan-Meier Survival Curves were presented to show the detrimental effect of dual activation of $\mathrm{HH}$ and Wnt signaling on outcome. Due to the small sample size, multi-variate analysis was not performed.

\section{Results}

Patient cohort. This study comprised a cohort of 36 tumors from women with histologically confirmed TNBC. A summary of clinical data is provided in Table I. This cohort included 21 patients with early stage disease (58\%, stage $1-2 \mathrm{~A})$ and 15 patients with late stage disease (42\%, stage $2 \mathrm{~B}-4$ ). The majority of patients presented with high grade tumors (grade $3,86 \%$ ) as is common in TNBC. Five patients $(14 \%)$ had grade 2 cancers.

Expression of stem cell pathways and relationship to clinical characteristics. Serial sections were stained for expression of SHH, Gli-1 and $\beta$-catenin. For SHH and Gli-1, slides were scored as having no expression (0), weak (1), moderate (2), or strong (3) tumor cell staining (Fig. 1; Table I). All samples showed cytoplasmic staining for SHH with $8(22 \%)$ showing weak, $20(56 \%)$ showing moderate, and 8 (22\%) showing strong staining (Fig. 1A; Table I). Expression of SHH was weakly correlated to tumor stage $(\mathrm{P}=0.044)$ (Table I). Cytoplasmic Gli-1 expression was observed in 35 (97\%) of the 36 samples, with 9 samples (25\%) showing weak staining, 14 samples (39\%) having moderate staining and 12 samples (33\%) having strong staining (Table I) (Fig. 1B). Nuclear Gli-1 expression was observed in 25 samples $(70 \%)$. Both cytoplasmic and nuclear Gli-1 expression correlated to tumor stage (Table I). 

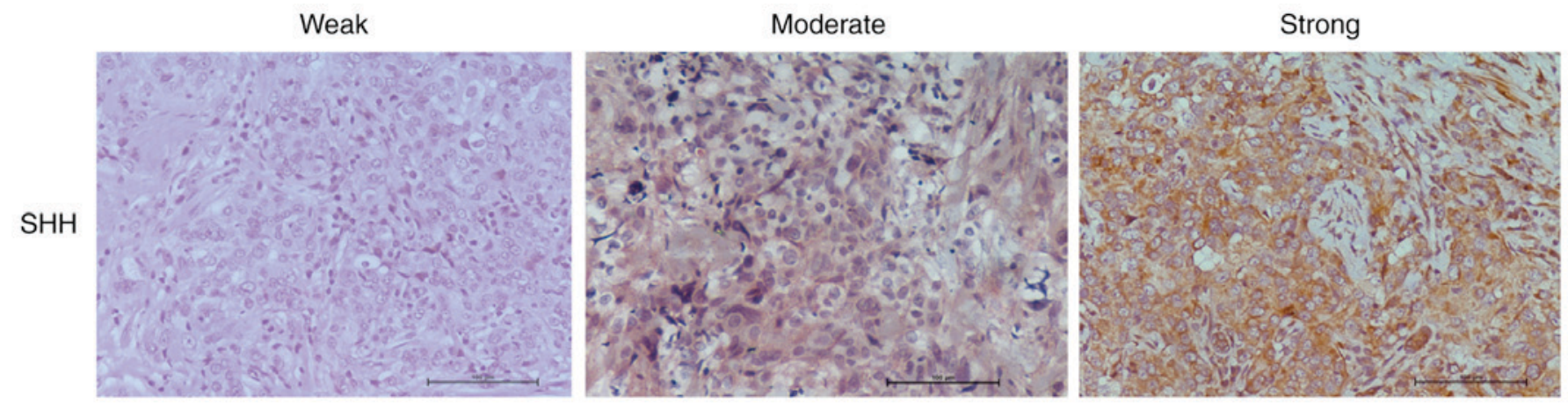

Figure 1. Immunohistochemical analysis of sonic Hedgehog (SHH) in triple negative breast cancer (TNBC) tumors. Representative images are shown of weak, moderate, and strong SHH staining. Images were acquired using a x20 magnification objective.

Gli-1
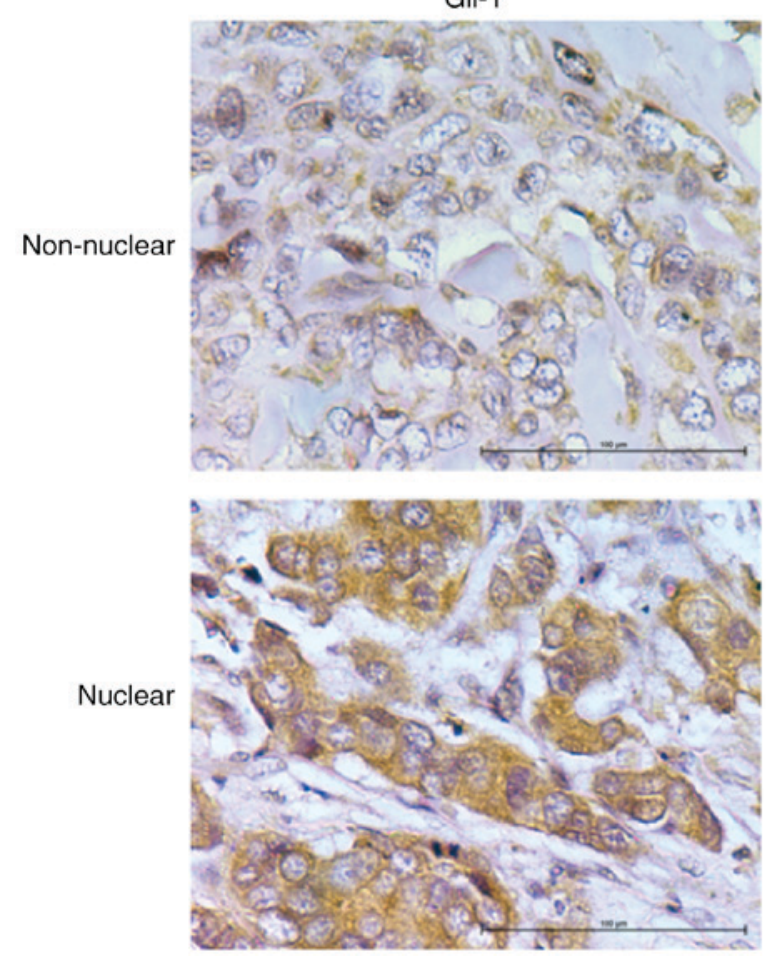

$\beta$-catenin
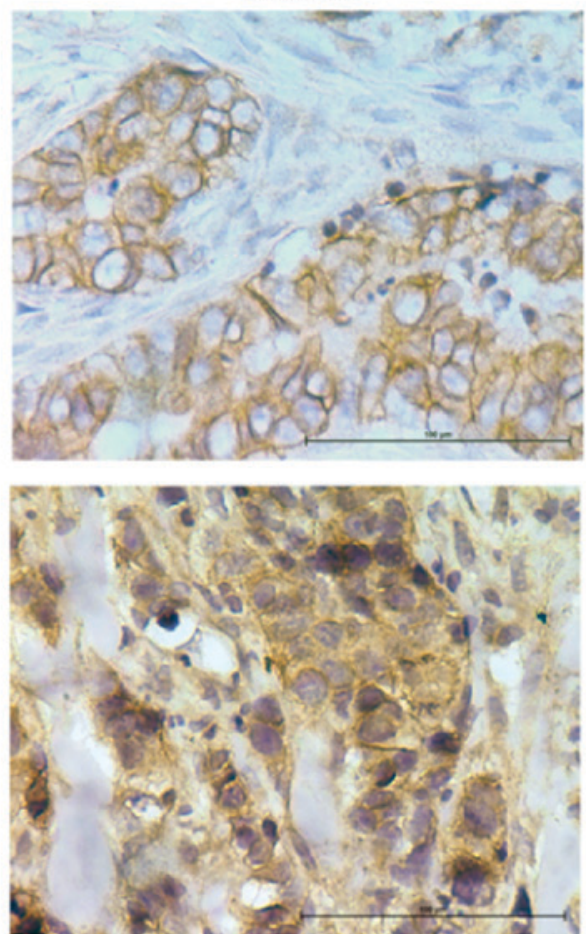

Figure 2. Immunohistochemical analysis of nuclear and non-nuclear glioma-associated oncogene homolog (Gli)-1 and $\beta$-catenin in triple negative breast cancer (TNBC) tumors. Images were acquired using a x40 magnification objective.

For $\beta$-catenin staining, slides were categorized as having membranous staining or cytoplasmic/nuclear staining (Fig. 2). Membranous $\beta$-catenin staining was observed in 13 samples (36\%). Nuclear/cytoplasmic staining was observed in 64\% of samples Cytoplasmic/nuclear $\beta$-catenin was highly correlated to tumor stage (Table I). There was no significant association with grade for any proteins examined (Table I). This is most likely due to the fact that most tumors $(n=31,86 \%)$ were grade 3 . This is consistent with the fact that most TNBC are high grade at diagnosis.

Association between HH and Wnt activation. SHH is significantly correlated to cytoplasmic expression of Gli-1 ( $\mathrm{P}<.001$, Table II). Interestingly, there was no association between nuclear Gli-1 and cytoplasmic SHH expression $(\mathrm{P}=0.291)$. Expression of both cytoplasmic and nuclear Gli-1 were correlated with tumor stage $(\mathrm{P}<.001)$ and each other $(\mathrm{P}<.001)$. Likewise, there was an association between cytoplasmic and nuclear $\beta$-catenin $(\mathrm{P}<.001)$ and both correlated to tumor stage $(\mathrm{P}<.003$, cytoplasmic, $\mathrm{P}<.001$ nuclear). SHH expression was significantly correlated to cytoplasmic/nuclear $\beta$-catenin $(\mathrm{P}<.001)$. Likewise, a significant correlation was observed between nuclear Gli-1 and nuclear $\beta$-catenin $(\mathrm{P}<.002)$ (Table II). This association remained when adjusting for tumor stage and grade.

Co-activation of $\mathrm{HH}$ and Wnt predict recurrence-free and overall survival. Independently, overexpression of SHH, Gli-1 and $\beta$-catenin, as well as nuclear localization of Gli-1 and $\beta$-catenin were associated with recurrence free and overall survival (Table I). To determine if dual activation of $\mathrm{HH}$ and Wnt pathways lead to a worse prognosis, patients were divided into three groups: those without activation of either pathway, 
Table II. Correlation of $\mathrm{HH}$ and WNT pathways in TNBC.

\begin{tabular}{llllll}
\hline Variables & & SHH & Gli-1 & Nuclear Gli & Nuclear Bcat \\
\hline SHH & Correlation coefficient & 1.000 & $0.598^{\mathrm{a}}$ & 0.181 & $0.520^{\mathrm{a}}$ \\
& P-value & & 0.000 & 0.291 & 0.001 \\
Gli-1 & Correlation coefficient & $0.598^{\mathrm{a}}$ & 1.000 & $0.585^{\mathrm{a}}$ & $0.649^{\mathrm{a}}$ \\
& P-value & 0.000 & & 0.000 & 0.000 \\
Nuclear Gli & Correlation coefficient & 0.181 & $0.585^{\mathrm{a}}$ & 1.000 & $0.506^{\mathrm{a}}$ \\
& P-value & 0.291 & 0.000 & & 0.002 \\
Nuclear/cytoplasmic & Correlation coefficient & $0.520^{\mathrm{a}}$ & $0.649^{\mathrm{a}}$ & $0.506^{\mathrm{a}}$ & 1.000 \\
$\beta$-catenin & P-value & 0.001 & 0.000 & 0.002 & \\
\hline
\end{tabular}

HH, Hedgehog; SHH, sonic HH; Gli-1, glioma-associated oncogene homolog-1; TNBC, triple negative breast cancer. ${ }^{\mathrm{a}} \mathrm{P}<0.05$.

A

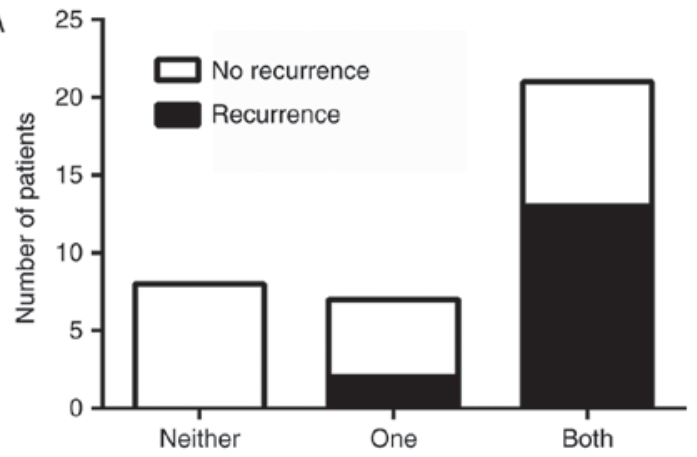

B

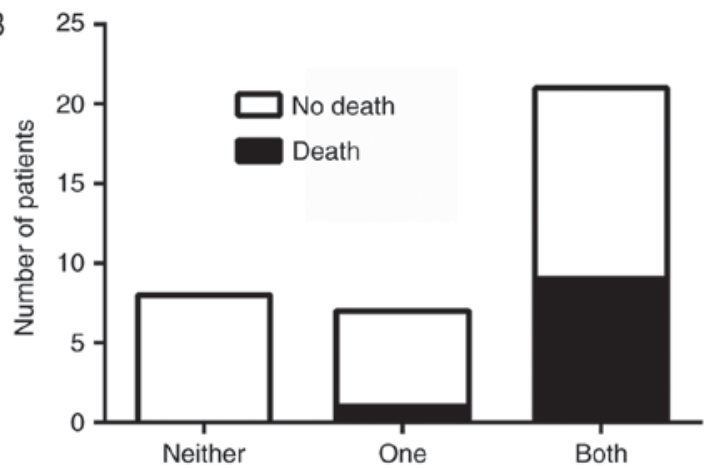

Figure 3. Graphical representation of the number of patients who (A) exhibited recurrence or (B) who had survived at the completion of this study in comparison to Hedgehog (HH) or Wnt activation. Patients were divided into three groups: No activation of $\mathrm{HH}$ or Wnt pathways, only one pathway activated or both pathways activated. Patients with dual activation of $\mathrm{HH}$ and canonical Wnt had poorer survival and were at greater risk of recurrence.

those with activation of only $\mathrm{HH}$ or Wnt, and those with activation of both pathways. There was an association between pathway activation and recurrence, $\chi^{2}(2)=11.11, \mathrm{P}=.004$, with those having activation of both $\mathrm{HH}$ and Wnt pathways being more likely to be in the later stage at diagnosis and at greater risk for recurrence (Fig. 3A). Likewise, there was an association between pathway activation and survival, $\chi^{2}(2)=6.75, \mathrm{P}=.034$, with longer survival observed in patients who lacked activation of both pathways (Fig. 3B). Progression free and overall survival time of the patients with dual activation of $\mathrm{HH}$ and Wnt pathways was significantly decreased (Fig. 4A and B).
A

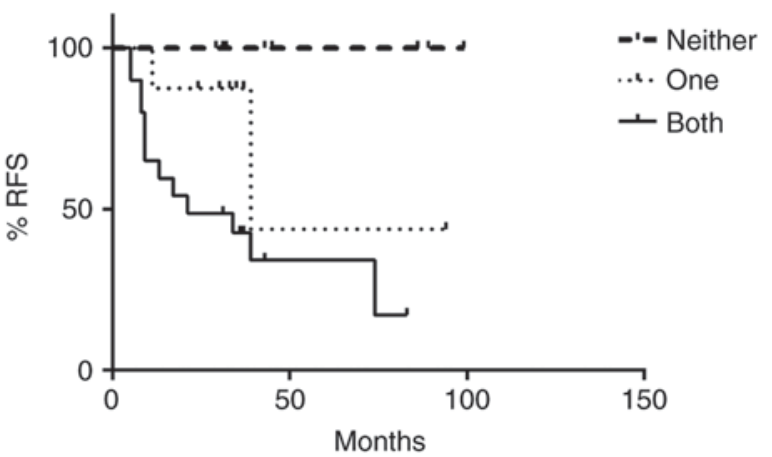

B

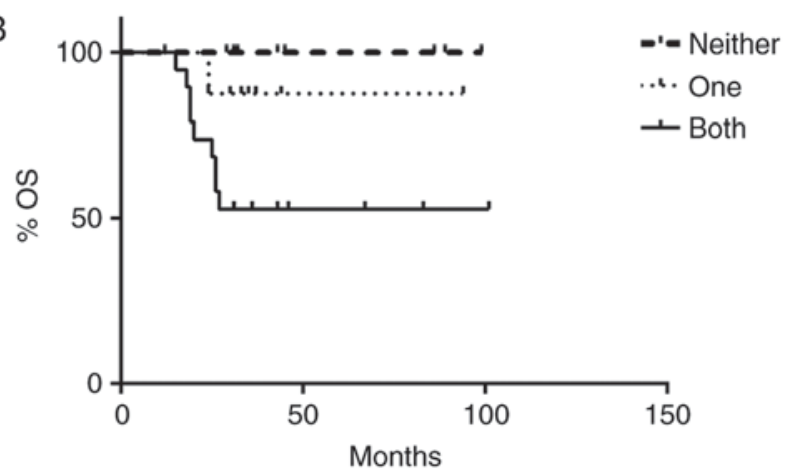

Figure 4. Kaplan-Meier (A) relapse-free survival (RFS) and (B) overall survival (OS) curves from women with triple negative breast cancer according to Hedgehog $(\mathrm{HH})$ and Wnt pathway activation. Patients were divided into three groups: no activation of $\mathrm{HH}$ or Wnt, only one pathway activated or both pathways activated. Co-activation of $\mathrm{HH}$ and canonical Wnt pathways resulted in poor patient outcome. Patients with both pathways activated had an increased risk for early recurrence and decreased overall survival when compared to patients with only one pathway activated. Patients with neither pathway activated had a $100 \%$ survival rate and no evidence of recurrence.

Patients who did not exhibit activation of $\mathrm{HH}$ or Wnt pathways had a $100 \%$ survival rate and no evidence of recurrence as of the end of this study. However, patients with both $\mathrm{HH}$ and Wnt pathways activated had a greater chance of early recurrence within the first three years compared to those with only one pathway activated, with greater than half of the patients exhibiting dual pathway activation relapsing. Patients with only one pathway activated had a higher risk of later recurrence than those without activation of either pathway, with greater than 
half of these patients relapsing after three years. Similarly, patients with both pathways activated had a lower survival rate (50\%) compared to patients with only one pathway activated $(87.5 \%)$ as of the end of this study (Fig. 4B).

\section{Discussion}

In this study we report activation of the $\mathrm{HH}$ and Wnt pathways in TNBC. Expression of SHH, GLI-1, $\beta$-catenin were independently associated to tumor stage. A significant correlation was observed between nuclear Gli-1 and nuclear $\beta$-catenin and our results indicate that co-activation of both $\mathrm{HH}$ and Wnt pathways is associated with shorter recurrence-free and overall survival times.

Our findings are consistent with previous studies that indicate an association between $\mathrm{HH}$ signaling and clinical outcome in breast cancer. Over expression of SMO and Gli-1 has been observed in TNBC compared to both normal breast tissue, mammary hyperplasia and ER+breast cancers (44). In this study, expression of Gli-1 was significantly correlated to tumor stage and lymphatic involvement. Alternatively, high expression of PTCH, and thereby inactivation of $\mathrm{HH}$ signaling, is associated with favorable prognosis (22). Moreover, a recent report in HER2+ breast cancer indicated that nuclear activation of Gli-1 was associated with an incomplete pathological response to neoadjuvant chemotherapy, and poorer survival in both hormone receptor positive and negative tumors (45). In addition, in inflammatory breast cancer, high expression of SHH is associated with poor prognosis (23). Interestingly, although expression levels of SHH and Gli-1 were associated with each other and tumor stage in our study, there was no significant association between SHH and nuclear Gli-1 in tumor cells. Although stromal staining was not included in our analysis, weak to moderate staining of SHH was observed in the stroma of several samples, and may contribute to paracrine activation of $\mathrm{HH}$ signaling. Additionally, non-canonical activation may be responsible for $\mathrm{HH}$ pathway activity in a subset of TNBC. Non-canonical activation of Gli-1 has been previously described in claudin-low subsets of breast cancer, and may be driven through a NF- $\mathrm{kB}$ dependent pathway (46).

Activation of Wnt signaling has also been associated to prognosis of TNBC (47). Overexpression of WNT ligands was found to predict recurrence in late stage TNBC (48). Wnt pathway activity is also associated to increased metastasis in TNBC. Using a classifier trained on $\beta$-catenin transfected mammary cells, in a meta-analysis of 11 studies and 1,878 breast cancer patients found Wnt activity associated primarily to TNBC compared to other subtypes of breast cancer (49). In addition, patients with high WNT activity were more likely to have increased risk of brain and lung metastasis.

Our data show increased nuclear activity of Gli-1 and $\beta$-catenin correlates with increasing tumor stage and dual activation of both is associated to shorter recurrence times and overall survival, suggesting a role in invasion and disease progression. Both pathways are involved in the regulation of genes critical for drug resistance $(50,51)$, EMT $(13,52)$ and differentiation $(53,54)$. Moreover, co-expression of $\mathrm{HH}$ and $\beta$-catenin was observed in poorly differentiated breast cancers compared to low grade and benign lesions (55). This expression correlated with a mesenchymal-like phenotype defined by expression of CD44 and vimentin, suggesting that simultaneous activation of $\mathrm{HH}$ and Wnt pathways may promote enhanced EMT and de-differentiation of breast cancer cells.

Although we observed significant expression of nuclear Gli-1 and nuclear $\beta$-catenin in TNBC samples, it is unknown if there is cross-talk between these pathways that results in increased activation of one or both pathways. Direct interactions of the $\mathrm{HH}$ and Wnt pathways have previously been reported. Transcriptome analysis of WNT3a responsive TNBC cell lines revealed Wnt target genes that are involved in the $\mathrm{HH}$ pathway signaling (56). Additionally, exogenous expression of constitutively nuclear $\beta$-catenin has been shown to increase activity of a Gli-1 reporter, suggesting that active Wnt signaling enhances Gli transcriptional activity (57). Likewise, inhibition of $\mathrm{HH}$ signaling with the SMO inhibitor cyclopamine has been reported to decrease $\beta$-catenin-TCF transcriptional activity in colon cancer lines (58). Further research is needed to determine the consequences of HH-Wnt interactions in breast cancer in relation to drug resistance and metastasis.

Our study is the first to report that co-activation of $\mathrm{HH}$ and Wnt pathways in clinical TNBC samples is associated to shorter recurrence times and decreased survival when compared to activation of only one of these pathways. Although this study was performed in a limited cohort of patients, our findings suggest that activation status of $\mathrm{HH}$ and Wnt pathways may provide a prognostic biomarker for patients at risk for treatment resistance and early recurrence and may be more valuable than independent analysis of either pathway. However, our limited sample size prevented multi-variant analysis to determine if co-activation of HH and WNT pathways occurs independent of tumor stage. These findings should be confirmed in subsequent studies with larger patient numbers.

\section{Acknowledgements}

This project was supported by the Delaware INBRE program, with a grant from the National Institute of General Medical Sciences-NIGMS (P20 GM103446) from the National Institutes of Health and the state of Delaware.

\section{References}

1. Carey LA, Perou CM, Livasy CA, Dressler LG, Cowan D, Conway K, Karaca G, Troester MA, Tse CK, Edmiston S, et al: Race, breast cancer subtypes, and survival in the carolina breast cancer study. JAMA 295: 2492-2502, 2006.

2. Foulkes WD, Stefansson IM, Chappuis PO, Bégin LR, Goffin JR, Wong N, Trudel M and Akslen LA: Germline BRCA1 mutations and a basal epithelial phenotype in breast cancer. J Natl Cancer Inst 95: 1482-1485, 2003.

3. Castiglioni F, Terenziani M, Carcangiu ML, Miliano R, Aiello P, Bertola L, Triulzi T, Gasparini P, Camerini T, Sozzi G, et al: Radiation effects on development of HER2-positive breast carcinomas. Clin Cancer Res 13: 46-51, 2007.

4. Dent R, Trudeau M, Pritchard KI, Hanna WM, Kahn HK, Sawka CA, Lickley LA, Rawlinson E, Sun P and Narod SA: Triple-negative breast cancer: Clinical features and patterns of recurrence. Clin Cancer Res 13: 4429-4434, 2007.

5. Hudis CA and Gianni L: Triple-negative breast cancer: An unmet medical need. Oncologist 16 (Suppl 1): S1-S11, 2011.

6. Jia Y and Xie J: Promising molecular mechanisms responsible for gemctiabine resistance in cancer. Genes Dis 2: 299-306, 2015.

7. Angeloni V, Tiberio P, Appierto V and Daidone MG: Implications of stemness-related signaling pathways in breast cancer response to therapy. Semin Cancer Biol 31: 43-51, 2015. 
8. Taipale $\mathrm{J}$ and Beachy PA: The hedgehog and Wnt signalling pathways in cancer. Nature 411: 349-354, 2001.

9. Reya $\mathrm{T}$ and Clevers $\mathrm{H}$ : Wnt signalling in stem cells and cancer. Nature 434: 843-850, 2005

10. Liu S, Dontu G, Mantle ID, Patel S, Ahn NS, Jackson KW, Suri $\mathrm{P}$ and Wicha MS: Hedgehog signaling and Bmi-1 regulate self-renewal of normal and malignant human mammary stem cells. Cancer Res 66: 6063-6071, 2006.

11. Takebe N, Harris PJ, Warren RQ and Ivy SP: Targeting cancer stem cells by inhibiting Wnt, notch, and hedgehog pathways. Nat Rev Clin Oncol 8: 97-106, 2011.

12. Clevers H, Loh KM and Nusse R: Stem cell signaling. An integral program for tissue renewal and regeneration: Wnt signaling and stem cell control. Science 346: 1248012, 2014.

13. Takebe N, Warren RQ and Ivy SP: Breast cancer growth and metastasis: Interplay between cancer stem cells, embryonic signaling pathways and epithelial-to-mesenchymal transition. Breast Cancer Res 13: 211,2011.

14. Flemban A and Qualtrough D: The potential role of hedgehog signaling in the luminal/basal phenotype of breast epithelia and in breast cancer invasion and metastasis. Cancers (Basel) 7: 1863-1884, 2015

15. Ingham PW and McMahon AP: Hedgehog signaling in animal development: Paradigms and principles. Genes Dev 15: 3059-3087, 2001

16. Lewis MT, Ross S, Strickland PA, Sugnet CW, Jimenez E, Scott MP and Daniel CW: Defects in mouse mammary gland development caused by conditional haploinsufficiency of Patched-1. Development 126: 5181-5193, 1999

17. Kubo M, Nakamura M, Tasaki A, Yamanaka N, Nakashima H, Nomura M, Kuroki S and Katano M: Hedgehog signaling pathway is a new therapeutic target for patients with breast cancer. Cancer Res 64: 6071-6074, 2004.

18. Moraes RC, Zhang X, Harrington N, Fung JY, Wu MF, Hilsenbeck SG, Allred DC and Lewis MT: Constitutive activation of smoothened (SMO) in mammary glands of transgenic mice leads to increased proliferation, altered differentiation and ductal dysplasia. Development 134: 1231-1242, 2007.

19. Hui M, Cazet A, Nair R, Watkins DN, O'Toole SA and Swarbrick A: The hedgehog signalling pathway in breast development, carcinogenesis and cancer therapy. Breast Cancer Res 15: 203, 2013.

20. Im S, Choi HJ, Yoo C, Jung JH, Jeon YW, Suh YJ and Kang CS Hedgehog related protein expression in breast cancer: Gli-2 is associated with poor overall survival. Korean J Pathol 47: 116-123, 2013.

21. Noman AS, Uddin M, Rahman MZ, Nayeem MJ, Alam SS, Khatun Z, Wahiduzzaman M, Sultana A, Rahman ML, Ali MY, et al: Overexpression of sonic hedgehog in the triple negative breast cancer: Clinicopathological characteristics of high burden breast cancer patients from Bangladesh. Sci Rep 6: 18830, 2016.

22. Wolf I, Bose S, Desmond JC, Lin BT, Williamson EA, Karlan BY and Koeffler HP: Unmasking of epigenetically silenced genes reveals DNA promoter methylation and reduced expression of PTCH in breast cancer. Breast Cancer Res Treat 105: 139-155, 2007.

23. Bièche I, Lerebours F, Tozlu S, Espie M, Marty M and Lidereau R: Molecular profiling of inflammatory breast cancer: Identification of a poor-prognosis gene expression signature. Clin Cancer Res 10: 6789-6795, 2004.

24. Polakis P: Wnt signaling and cancer. Genes Dev 14: 1837-1851, 2000.

25. Nelson WJ and Nusse R: Convergence of Wnt, beta-catenin, and cadherin pathways. Science 303: 1483-1487, 2004.

26. Duchartre Y, Kim YM and Kahn M: The Wnt signaling pathway in cancer. Crit Rev Oncol Hematol 99: 141-149, 2016.

27. Katoh M and Katoh M: WNT signaling pathway and stem cell signaling network. Clin Cancer Res 13: 4042-4045, 2007.

28. Jamieson C, Sharma M and Henderson BR: Targeting the $\beta$-catenin nuclear transport pathway in cancer. Semin Cancer Biol 27: 20-29, 2014.

29. Clevers H: Wnt/beta-catenin signaling in development and disease. Cell 127: 469-480, 2006.

30. Jönsson M, Borg A, Nilbert $M$ and Andersson T: Involvement of adenomatous polyposis coli (APC)/beta-catenin signalling in human breast cancer. Eur J Cancer 36: 242-248, 2000.

31. Lin SY, Xia W, Wang JC, Kwong KY, Spohn B, Wen Y, Pestell RG and Hung MC: Beta-catenin, a novel prognostic marker for breast cancer: Its roles in cyclin D1 expression and cancer progression. Proc Natl Acad Sci USA 97: 4262-4266, 2000
32. King TD, Suto MJ and Li Y: The Wnt/ $\beta$-catenin signaling pathway: A potential therapeutic target in the treatment of triple negative breast cancer. J Cell Biochem 113: 13-18, 2012.

33. Hatsell S, Rowlands T, Hiremath M and Cowin P: Beta-catenin and Tcfs in mammary development and cancer. J Mammary Gland Biol Neoplasia 8: 145-158, 2003.

34. Huguet EL, McMahon JA, McMahon AP, Bicknell R and Harris AL: Differential expression of human Wnt genes 2, 3, 4 , and $7 \mathrm{~B}$ in human breast cell lines and normal and disease states of human breast tissue. Cancer Res 54: 2615-2621, 1994.

35. Benhaj K, Akcali KC and Ozturk M: Redundant expression of canonical Wnt ligands in human breast cancer cell lines. Oncol Rep 15: 701-707, 2006.

36. Nagahata T, Shimada T, Harada A, Nagai H, Onda M, Yokoyama S, Shiba T, Jin E, Kawanami O and Emi M: Amplification, up-regulation and over-expression of DVL-1, the human counterpart of the Drosophila disheveled gene, in primary breast cancers. Cancer Sci 94: 515-518, 2003.

37. Bànkfalvi A, Terpe HJ, Breukelmann D, Bier B, Rempe D, PschadkaG,Krech R,LellèRJ and BoeckerW:Immunophenotypic and prognostic analysis of E-cadherin and beta-catenin expression during breast carcinogenesis and tumour progression: A comparative study with CD44. Histopathology 34: 25-34, 1999.

38. Karayiannakis AJ, Nakopoulou L, Gakiopoulou H, Keramopoulos A, Davaris PS and Pignatelli M: Expression patterns of beta-catenin in in situ and invasive breast cancer. Eur J Surg Oncol 27: 31-36, 2001.

39. Wong SC, Lo SF, Lee KC, Yam JW, Chan JK and Wendy Hsiao WL: Expression of frizzled-related protein and Wnt-signalling molecules in invasive human breast tumours. J Pathol 196: 145-153, 2002.

40. Yang SH, Andl T, Grachtchouk V, Wang A, Liu J, Syu LJ, Ferris J, Wang TS, Glick AB, Millar SE and Dlugosz AA: Pathological responses to oncogenic hedgehog signaling in skin are dependent on canonical Wnt/beta3-catenin signaling. Nat Genet 40: 1130-1135, 2008

41. He J, Sheng T, Stelter AA, Li C, Zhang X, Sinha M, Luxon BA and Xie J: Suppressing Wnt signaling by the hedgehog pathway through sFRP-1. J Biol Chem 281: 35598-35602, 2006.

42. Schneider FT, Schänzer A, Czupalla CJ, Thom S, Engels K, Schmidt MH, Plate KH and Liebner S: Sonic hedgehog acts as a negative regulator of $\{$ beta\}-catenin signaling in the adult tongue epithelium. Am J Pathol 177: 404-414, 2010.

43. Opdenaker LM, Arnold KM, Pohlig RT, Padmanabhan JS, Flynn DC and Sims-Mourtada J: Immunohistochemical analysis of aldehyde dehydrogenase isoforms and their association with estrogen-receptor status and disease progression in breast cancer. Breast Cancer (Dove Med Press) 6: 205-209, 2014.

44. Tao Y, Mao J, Zhang Q and Li L: Overexpression of hedgehog signaling molecules and its involvement in triple-negative breast cancer. Oncol Lett 2: 995-1001, 2011.

45. Liu S, Duan X, Xu L, Ye J, Cheng Y, Liu Q, Zhang H, Zhang S, Zhu S, Li T and Liu Y: Nuclear Gli1 expression is associated with pathological complete response and event-free survival in HER2-positive breast cancer treated with trastuzumab-based neoadjuvant therapy. Tumour Biol 37: 4873-4881, 2016.

46. Colavito SA, Zou MR, Yan Q, Nguyen DX and Stern DF: Significance of glioma-associated oncogene homolog 1 (GLI1) expression in claudin-low breast cancer and crosstalk with the nuclear factor kappa-light-chain-enhancer of activated B cells (NFאB) pathway. Breast Cancer Res 16: 444, 2014.

47. Xu WH, Liu ZB, Yang C, Qin W and Shao ZM: Expression of dickkopf-1 and beta-catenin related to the prognosis of breast cancer patients with triple negative phenotype. PLoS One 7: e37624, 2012

48. Tsai CH, Chiu JH, Yang CW, Wang JY, Tsai YF, Tseng LM, Chen WS and Shyr YM: Molecular characteristics of recurrent triple-negative breast cancer. Mol Med Rep 12: 7326-7334, 2015.

49. Dey N, Barwick BG, Moreno CS, Ordanic-Kodani M, Chen Z, Oprea-Ilies G, Tang W, Catzavelos C, Kerstann KF, Sledge GW Jr, et al: Wnt signaling in triple negative breast cancer is associated with metastasis. BMC Cancer 13: 537, 2013.

50. Sims-Mourtada J, Izzo JG, Ajani J and Chao KS: Sonic hedgehog promotes multiple drug resistance by regulation of drug transport. Oncogene 26: 5674-5679, 2007.

51. Xia Z, Guo M, Liu H, Jiang L, Li Q, Peng J, Li JD, Shan B, Feng P and Ma H: CBP-dependent Wnt/ $\beta$-catenin signaling is crucial in regulation of MDR1 transcription. Curr Cancer Drug Targets 15: 519-532, 2015. 
52. Micalizzi DS, Farabaugh SM and Ford HL: Epithelial-mesenchymal transition in cancer: Parallels between normal development and tumor progression. J Mammary Gland Biol Neoplasia 15: 117-134, 2010.

53. Alexander CM, Goel S, Fakhraldeen SA and Kim S: Wnt signaling in mammary glands: Plastic cell fates and combinatorial signaling. Cold Spring Harb Perspect Biol 4: pii: a008037, 2012.

54. Kakarala M and Wicha MS: Implications of the cancer stem-cell hypothesis for breast cancer prevention and therapy. J Clin Oncol 26: 2813-2820, 2008.

55. Scimeca M, Antonacci C, Colombo D, Bonfiglio R, Buonomo OC and Bonanno E: Emerging prognostic markers related to mesenchymal characteristics of poorly differentiated breast cancers. Tumour Biol 37: 5427-5435, 2016.
56. Maubant S, Tesson B, Maire V, Ye M, Rigaill G, Gentien D, Cruzalegui F, Tucker GC, Roman-Roman S and Dubois T: Transcriptome analysis of Wnt3a-treated triple-negative breast cancer cells. PLoS One 10: e0122333, 2015.

57. Maeda O, Kondo M, Fujita T, Usami N, Fukui T, Shimokata K, Ando T, Goto H and Sekido Y: Enhancement of GLI1-transcriptional activity by beta-catenin in human cancer cells. Oncol Rep 16: 91-96, 2006.

58. Qualtrough D, Rees P, Speight B, Williams AC and Paraskeva C: The hedgehog inhibitor cyclopamine reduces $\beta$-catenin-Tcf transcriptional activity, induces E-cadherin expression, and reduces invasion in colorectal cancer cells. Cancers (Basel) 7: 1885-1899, 2015. 